\title{
The Dynamic Behaviour of the Competing Species with Linear and Holling Type II Functional Responses by the Second Competitor
}

\author{
Alemu Geleta Wedajo ${ }^{\mathrm{i}}$, N. Phani Kumar ${ }^{\mathrm{ii}}$, K.V.L.N.Acharyulu ${ }^{\mathrm{iii}} \&$ Boka Kumsa $^{\mathrm{iv}}$

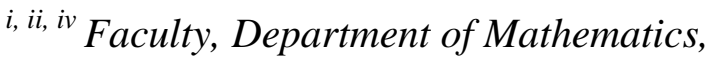 \\ College of Natural and Computational Sciences, Nekemte, Ethiopia. \\ ${ }^{i i i}$ Associate Professor, Department of Mathematics, Bapatla Engineering College, \\ Andhra Pradesh, India. \\ kvlna@yahoo.com
}

\begin{abstract}
The purpose of this paper is to discuss the existence of continuous models for interacting populations by using differential equations. The preliminaries contain all the possible efforts to facilitate the understanding of population dynamics; competition Models, for which a qualitative theory is available and also some numerical illustration will be given. The paper contains three main topics; the first one is basic Lotka-Volterra competition model, the second one presents the competition model with carrying capacity in which both are with the same linear functional response. The third one is devoted to study the qualitative behavior of a competition model with Holling type II functional response which shows two species competing with each other in linear functional response. In each topic, we shall derive the biological models, and then we do the nondimensional analysis to reduce the model to a simple model with fewer parameters. Based on these three ideas of qualitative study of competition model, the results, implications and observations along with conclusions are exemplified.
\end{abstract}

Keywords: Non-dimensionalization, Equilibrium (critical) points, community matrix, competing species

\section{Introduction}

Competition is an interaction between two species that is mutually detrimental [5]. Competition between organisms occurs in case of limited supply of essential resource, such as food, in case of animals and water, nutrients and light in the case of plants $[2,6]$. In many cases in this paper, competitor I and competitor II will not depend explicitly on time, i.e. $M_{1}(t)=f\left(M_{1}, M_{2}\right)$ and $M_{2}(t)=g\left(M_{1}, M_{2}\right)$. Such systems are called autonomous. Thus the vector field $(f, g)$ giving the velocity of a point $\left(M_{1}, M_{2}\right)$ is fixed in time. To study such systems we can construct the phase plane, i.e. a picture of the solution trajectories mapped out by points $\left(M_{1}(t), M_{2}(t)\right)$ as $t$ varies over $(-\infty,+\infty)$. In particular we identify the steady state populations and study the qualitative behavior of the system $[1,3,4]$.

\section{Assumptions and Model Formulation}

In this paper, we describe the competition model. To develop this model the assumptions have been made.

i. $\quad M_{1}(t)$ and $M_{2}(t)$ denote the density of competitor I and competitor II respectively at any instant of time $t$ subject to the non-negative initial conditions

$$
M_{1}(0)=M_{10} \geq 0 \text { and } M_{2}(0)=M_{20} \geq 0 \text {. }
$$


ii. The parameters $r_{i}$ and $k_{i}, \mathrm{i}=1,2$ be the intrinsic birth rates and the environmental carrying capacities of competitor I and competitor II respectively, and it is also assumed that the growth of the competitors is logistic.

iii. The positive parameters $b$ and $d$ measure the strength of inter specific competition of the two species and the parameters $a$ and $b$ assumes that two species with respective populations $M_{1}$ and $M_{2}$ each grow logistically in the absence of the other. Competitor I and competitor II compete in a linear response type I, $b M_{1}$.

iv. The term $\frac{b M_{1}}{1+b h M_{1}}$ represents the functional response for competition of competitor I by competitor II. This functional response is called Holling type II functional response which represents the rate at which competitor II competes competitor I, in addition to that the competitor II needs on average $\mathrm{h}$ time units to compete each single number of competitor I. The type II functional response is hence based on the assumption that at very high competitor I densities, competitor II becomes limited by their handling time $\mathrm{h}$. Indeed, for $M_{1} \rightarrow \infty$ the limit of the functional response equals the inverse of handling time $\frac{1}{h}$.

Under the above mentioned assumptions, we describe the competitor I- competitor II model by the following two differential equations.

\section{Type-I}

We start with the assumption that two species with respective populations $M_{1}$ and $M_{2}$ each grow logistically in the absence of the other, as described by the following uncoupled logistic equations:

$$
\begin{aligned}
& \frac{d M_{1}}{d t}=a M_{1}-b M_{1} M_{2} \\
& \frac{d M_{1}}{d t}=c M_{2}-d M_{1} M_{2}
\end{aligned}
$$

\subsection{Equilibrium Points or Steady States}

Next, we may obtain the system's equilibrium points by finding values of $M_{1}$ and $M_{2}$ for which $\frac{d M_{1}}{d t}=\frac{d M_{2}}{d t}=0$ is satisfied.

Hence the system (1.1) possesses the following steady states:

i. The trivial steady state $E_{T}=(0,0)$

ii. The co-existence steady state $E_{c}=\left(\frac{c}{d}, \frac{a}{b}\right)$ which is the element of the first quadrant of $M_{1}$ versus $M_{2}$ coordinate axis.

\subsection{Linearization of the Given System}

Next we linearize the given system at the equilibrium points. To do this we let

$$
\begin{aligned}
& \frac{d M_{1}}{d t}=a M_{1}-b M_{1} M_{2}=f\left(M_{1}, M_{2}\right) \\
& \frac{d M_{2}}{d t}=c M_{2}-d M_{1} M_{2}=g\left(M_{1}, M_{2}\right)
\end{aligned}
$$

Thus the Jacobian matrix or variational matrix is given by:

$$
J=\left[\begin{array}{ll}
\frac{\partial f\left(M_{1}, M_{2}\right)}{\partial M_{1}} & \frac{\partial f\left(M_{1}, M_{2}\right)}{\partial M_{2}} \\
\frac{\partial g\left(M_{1}, M_{2}\right)}{\partial M_{1}} & \frac{\partial g\left(M_{1}, M_{2}\right)}{\partial M_{2}}
\end{array}\right]=\left[\begin{array}{cc}
a-b M_{2} & -b M_{1} \\
-d M_{2} & c-d M_{1}
\end{array}\right]
$$


Now evaluating the Jacobian matrix or variational matrix at each of the equilibrium points above $(0,0)$ and $\left(\frac{\boldsymbol{c}}{\boldsymbol{d}}, \frac{\boldsymbol{a}}{\boldsymbol{b}}\right)$ we get $J=\left[\begin{array}{ll}a & 0 \\ 0 & c\end{array}\right]$ and $J=\left[\begin{array}{cc}0 & \frac{-b c}{d} \\ \frac{-a d}{b} & 0\end{array}\right]$ respectively.

\subsection{Stability Analysis}

Now, with this information on hand, we may continue our analysis by determining the stability of each equilibrium point, and viewing the qualitative behavior of each case with the help of Phase plane analysis which is one of the most important techniques for studying the behavior of nonlinear systems, since there is usually no analytical solution for a nonlinear system. [1, 4].

Theorem 1.1: The equilibrium point $(0,0)$ is unstable node.

Proof: At a critical point $(0,0)$ the characteristic polynomial is given by:

$$
P(\lambda)=\lambda^{2}-(a+c) \lambda+a c
$$

Hence the corresponding Eigen values are obtained by letting $P(\lambda)=0$ which yield $\lambda_{1}=a$ and $\lambda_{2}=c$. Since $\mathrm{a}$ and $\mathrm{c}$ are assumed to be constant positive real numbers such that the equilibrium point $(0,0)$ is unstable node.

Theorem 1.2: The equilibrium point $\left(\frac{c}{d}, \frac{a}{b}\right)$ is a saddle point.

Proof: At an equilibrium point $\left(\frac{c}{d}, \frac{a}{b}\right)$ the characteristic polynomial is given by

$$
P(\lambda)=\lambda^{2}-a c
$$

Hence the corresponding Eigen values are obtained by letting $P(\lambda)=0$ which yields $\lambda_{1}=\sqrt{a c}$ and $\lambda_{2}=-\sqrt{a c}$. Since a and $\mathrm{c}$ are assumed to be constant positive real numbers such that $a c>0$ which implies $\sqrt{a c}$ is defined resulting in, the equilibrium point $\left(\frac{c}{d}, \frac{a}{b}\right)$ is a saddle point.

\subsection{Numerical Illustration}

In this section, the qualitative behavior of the proposed uncoupled logistic model (1.1) has been discussed using Phase plane diagram. Due to unavailability of real data of all parameters associated with the model, the following parametric values are used to analyze the given model qualitatively by using Phase plane diagram.

$$
a=4, b=2, c=6, d=3,-10 \leq M_{1} \leq 10 \text { and }-10 \leq M_{2} \leq 10
$$

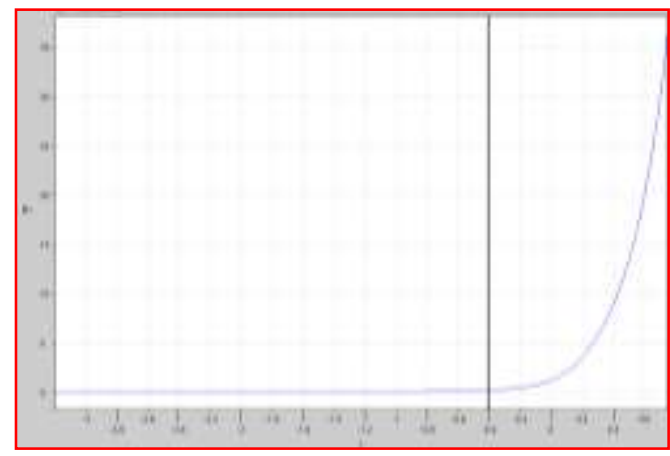

Figure 1.1. Competitor I Versus Time Graph

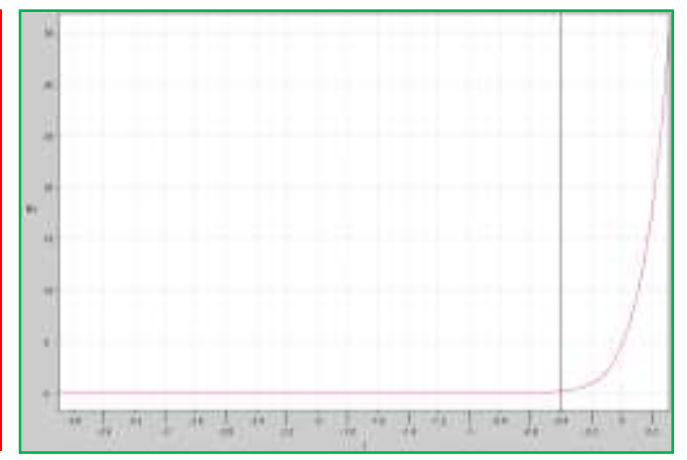

Figure 1.2. Competitor II Versus Time Graph 
The phase plane diagram for uncoupled logistic competition model:

with the chosen parameters is given by

$$
\begin{aligned}
& \frac{d M_{1}}{d t}=a M_{1}-b M_{1} M_{2}=f\left(M_{1}, M_{2}\right) \\
& \frac{d M_{2}}{d t}=c M_{2}-d M_{1} M_{2}=g\left(M_{1}, M_{2}\right)
\end{aligned}
$$

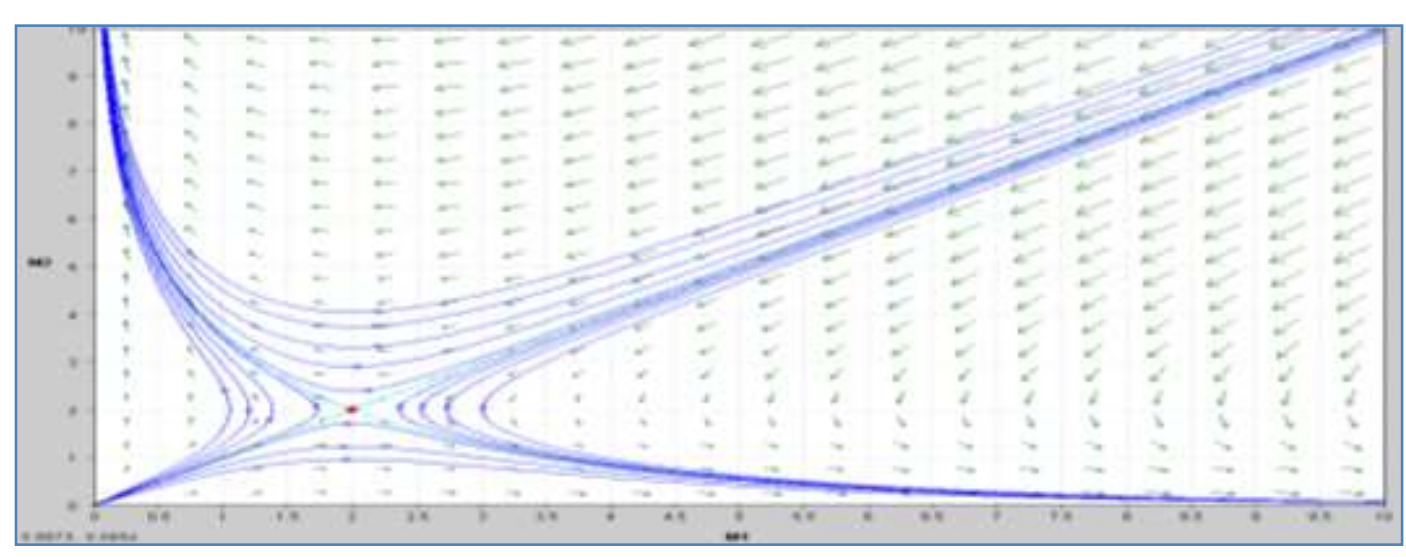

Figure 1.3. Phase diagram for $\frac{d M_{1}}{d t}=a M_{1}-b M_{1} M_{2}$ and $\frac{d M_{2}}{d t}=c M_{2}-d M_{1} M_{2}$

\subsection{Observation}

We observed that the schematic phase trajectories near the steady states for the dynamic behavior of competing populations satisfying the model (1.1) for the parameters chosen there is no stable steady state in which all trajectories tend to it.

\section{Type-II}

Now incorporating the interacting logistic model, we will begin with a classical model of competition based on the work of Lotka and Volterra where in two species are assumed to have a hibitary effect on each other. The logistic equation for two interacting species $\mathrm{M}_{1}$ and $\mathrm{M}_{2}$, that is, when the species compete with each other (for nesting sites, food, etc.), the interspecific competition is detrimental to both species per capita growth rates. The simplest model is to say that the per capita growth rates decrease linearly with the density of the other species.

The competition equation in this case is:

$$
\frac{d M_{1}}{d t}=r_{1} M_{1}\left(1-\frac{M_{1}}{k_{1}}\right)-b M_{1} M_{2}
$$

$$
\frac{d M_{2}}{d t}=r_{2} M_{2}\left(1-\frac{M_{2}}{k_{2}}\right)-d M_{1} M_{2}
$$

In equation (1.2), $M_{1}$ and $M_{2}$ species grows logistically and they compete in a linear functional response, $b M_{1}$. 


\subsection{Non-dimensionalization}

To ease calculations we first non-dimensionalize by setting

$$
u_{i}=\frac{M_{i}}{k_{i}} \text { for } i=1,2
$$

we also introduce a dimensionless time $\tau=r_{1} t$ and set $r=\frac{r_{2}}{r_{1}}$

Therefore the simplest set of equations with fewer parameters is then given by:

$$
\begin{aligned}
& \frac{d u_{1}}{d \tau}=u_{1}\left[1-u_{1}-a_{12} u_{2}\right] \\
& \frac{d u_{2}}{d \tau}=r u_{2}\left[1-u_{2}-a_{21} u_{1}\right]
\end{aligned}
$$

where $a_{12}=\frac{b k_{2}}{r_{1}}$ and $a_{21}=\frac{d k_{1}}{r_{2}}$

Next, we may obtain the system's equilibrium (critical) points by finding values of $u_{1}$ and $u_{2}$ for which $\frac{d u_{1}}{d \tau}=\frac{d u_{2}}{d \tau}=0$ is satisfied.

Thus the equilibrium points are $(0,0),(0,1),(1,0)$ and $\left(\frac{1-a_{12}}{1-a_{12} a_{21}}, \frac{1-a_{21}}{1-a_{12} a_{21}}\right)$

\subsection{Linearization of the Given Logistic Competition Model System}

To linearize the given system at each of the equilibrium points we let:

$$
\begin{aligned}
& \frac{d u_{1}}{d \tau}=u_{1}\left(1-u_{1}-a_{12} u_{2}\right)=f\left(u_{1}, u_{2}\right) \\
& \frac{d u_{2}}{d \tau}=r u_{2}\left(1-u_{2}-a_{21} u_{1}\right)=g\left(u_{1}, u_{2}\right)
\end{aligned}
$$

Hence the Jacobian or community matrix is:

$$
J=\left[\begin{array}{ll}
\frac{\partial f\left(u_{1}, u_{2}\right)}{\partial u_{1}} & \frac{\partial f\left(u_{1}, u_{2}\right)}{\partial u_{2}} \\
\frac{\partial g\left(u_{1}, u_{2}\right)}{\partial u_{1}} & \frac{\partial g\left(u_{1}, u_{2}\right)}{\partial u_{2}}
\end{array}\right]=\left[\begin{array}{cc}
1-2 u_{1}-a_{12} u_{2} & -a_{12} u_{1} \\
-r a_{21} u_{2} & r-2 r u_{2}-r a_{21} u_{1}
\end{array}\right]
$$

Theorem 1.3: The equilibrium point $(0,0)$ is a source or repller.

Proof: Evaluating the Jacobian matrix at an equilibrium point $(0,0)$ we obtain $J=\left[\begin{array}{ll}1 & 0 \\ 0 & r\end{array}\right]$ The corresponding characteristic equation is:

$$
P(\lambda)=\lambda^{2}-(1+r) \lambda+r
$$

Hence the corresponding Eigen values are $\lambda_{1}=1$ and $\lambda_{2}=r$. Since $r$ is a positive real number, the equilibrium point $(0,0)$ is a source or repller.//

Theorem 1.4: The equilibrium point $(0,1)$ is a stable node or attractor or sink.

Proof: The corresponding Jaccobian matrix is at an equilibrium point $(0,1)$ is $J=$ $\left[\begin{array}{cc}-1 & 0 \\ -r a_{21} & -r\end{array}\right]$

Thus the characteristic equation is:

$$
P(\lambda)=\lambda^{2}+(1+r) \lambda+r
$$

Hence the Eigen values are $\lambda_{1}=-1$ and $\lambda_{2}=-r$. Since $r$ is a positive real number, both the Eigen values are negative yielding the equilibrium point $(0,1)$ is a stable node or attractor or sink.// 
Theorem 1.5: The equilibrium point $(1,0)$ is a nodal sink.

Proof: At an equilibrium point $(1,0)$ we obtain a Jaccobian matrix $J=\left[\begin{array}{cc}-1 & a_{12} \\ 0 & r-r a_{21}\end{array}\right]$ with the corresponding Eigen values and Eigen vectors:

$$
\lambda_{1}=-1, \quad\left[\begin{array}{c}
u_{11} \\
0
\end{array}\right] \text { and } \lambda_{2}=r-a_{21} r, \quad\left[\begin{array}{c}
a_{12} \\
-1-r+r a_{21}
\end{array}\right] .
$$

Since $r$ is a positive real number and $r \neq 1$ such that $r\left(1-a_{21}\right)<0$ yielding the equilibrium point $(1,0)$ is a nodal sink.

The last equilibrium point $\left(u_{1}{ }^{*}, u_{2}{ }^{*}\right)=\left(\frac{1-a_{12}}{1-a_{12} a_{21}}, \frac{1-a_{21}}{1-a_{12} a_{21}}\right)$ is only feasible i.e., nonnegative populations when either:

1. $\mathrm{a}_{12}<1$ and $\mathrm{a}_{21}<1$, since then also $1-\mathrm{a}_{12} \mathrm{a}_{21}>0$

2. $\mathrm{a}_{12}>1$ and $\mathrm{a}_{21}>1$, since then also $1-\mathrm{a}_{12} \mathrm{a}_{21}<0$

In this case, a critical point exists interior to the positive quadrant, meaning the two species coexist.

The Jacobian matrix at this equilibrium point is:

$J$

$$
\begin{gathered}
=\left[\begin{array}{cc}
1-2\left(\frac{a_{12}-1}{a_{12} a_{21}-1}\right)-a_{12}\left(\frac{a_{21}-1}{a_{12} a_{21}-1}\right) & -a_{12}\left(\frac{a_{12}-1}{a_{12} a_{21}-1}\right) \\
-r a_{21}\left(\frac{a_{21}-1}{a_{12} a_{21}-1}\right) & r\left(1-2\left(\frac{a_{21}-1}{a_{12} a_{21}-1}\right)-a_{21}\left(\frac{a_{12}-1}{a_{12} a_{21}-1}\right)\right)
\end{array}\right] \\
=\left[\begin{array}{cc}
\frac{a_{12} a_{21}-1-2 a_{12}+2-a_{12} a_{21}+a_{12}}{a_{12} a_{21}-1} & \frac{-a^{2}{ }_{12}+a_{12}}{a_{12} a_{21}-1} \\
\frac{-r a^{2}{ }_{21}+r a_{21}}{a_{12} a_{21}-1} & r\left(\frac{a_{12} a_{21}-1-2 a_{21}+2-a_{21} a_{12}+a_{21}}{a_{12} a_{21}-1}\right)
\end{array}\right] \\
=\left[\begin{array}{cc}
\frac{1-a_{12}}{a_{12} a_{21}-1} & \frac{-a^{2}{ }_{12}+a_{12}}{a_{12} a_{21}-1} \\
\frac{-r a^{2}{ }_{21}+r a_{21}}{a_{12} a_{21}-1} & r\left(\frac{1-a_{21}}{a_{12} a_{21}-1}\right)
\end{array}\right]=\left[\begin{array}{cc}
-u_{1}{ }^{*} & a_{12} u_{1}{ }^{*} \\
-r u_{2}{ }^{*} a_{21} & -r u_{2}{ }^{*}
\end{array}\right]
\end{gathered}
$$

The characteristic polynomial corresponding to $\mathrm{J}$ is given by:

$$
\lambda^{2}-T \lambda+D=0
$$

where $\mathrm{T}$ and $\mathrm{D}$ are respectively trace and determinant of $\mathrm{J}$.

As discussed above, the equilibrium point $\left(u_{1}{ }^{*}, u_{2}{ }^{*}\right)=\left(\frac{1-a_{12}}{1-a_{12} a_{21}}, \frac{1-a_{21}}{1-a_{12} a_{21}}\right)$ is meaningful only if $u_{1}{ }^{*}>0$ and $u_{2}{ }^{*}>0$.

Since $u_{1}{ }^{*}>0$ and $u_{2}{ }^{*}>0$, then the trace of $\mathrm{J}$ is negative. So the sum of the Eigen values is negative.

From determinant of $\mathrm{J}, D=r u_{1}{ }^{*} u_{2}{ }^{*}\left(1-a_{12} a_{21}\right)$ :

The equilibrium point $\left(\frac{1-a_{12}}{1-a_{12} a_{21}}, \frac{1-a_{21}}{1-a_{12} a_{21}}\right)$ is:

i) Stable attractor if $a_{12}<1$ and $a_{21}<1$, since then also $a_{12} a_{21}<1$.

ii) A saddle point if $a_{12}>1$ and $a_{21}>1$, since then also $a_{12} a_{21}>1$.

\subsection{Numerical Illustration}

For the set of parametric values $r=2, a_{12}=3, a_{21}=5,-5 \leq u_{1} \leq 5$ and $-5 \leq$ $u_{2} \leq 5$ we observe the isoclines and phase plane portrait as follows.

Therefore for these cases we obtain the following isoclines for the system:

$\frac{d u_{1}}{d \tau}=u_{1}\left(1-u_{1}-a_{12} u_{2}\right) \& \frac{d u_{2}}{d \tau}=r u_{2}\left(1-u_{2}-a_{21} u_{1}\right)$ 


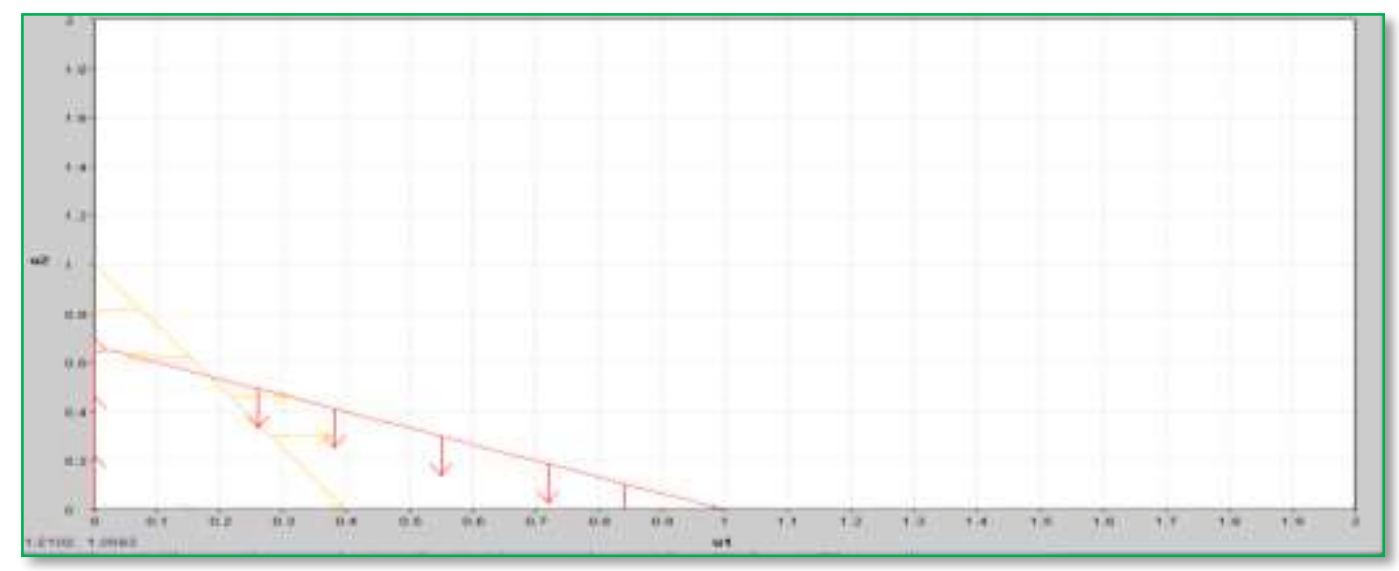

Figure 1.4. The Isoclines for the System $\frac{d u_{1}}{d \tau}=u_{1}\left(1-u_{1}-a_{12} u_{2}\right), \frac{d u_{2}}{d \tau}=$ $r u_{2}\left(1-u_{2}-a_{21} u_{1}\right)$

for $a_{12}>1$ and $a_{21}>1$.

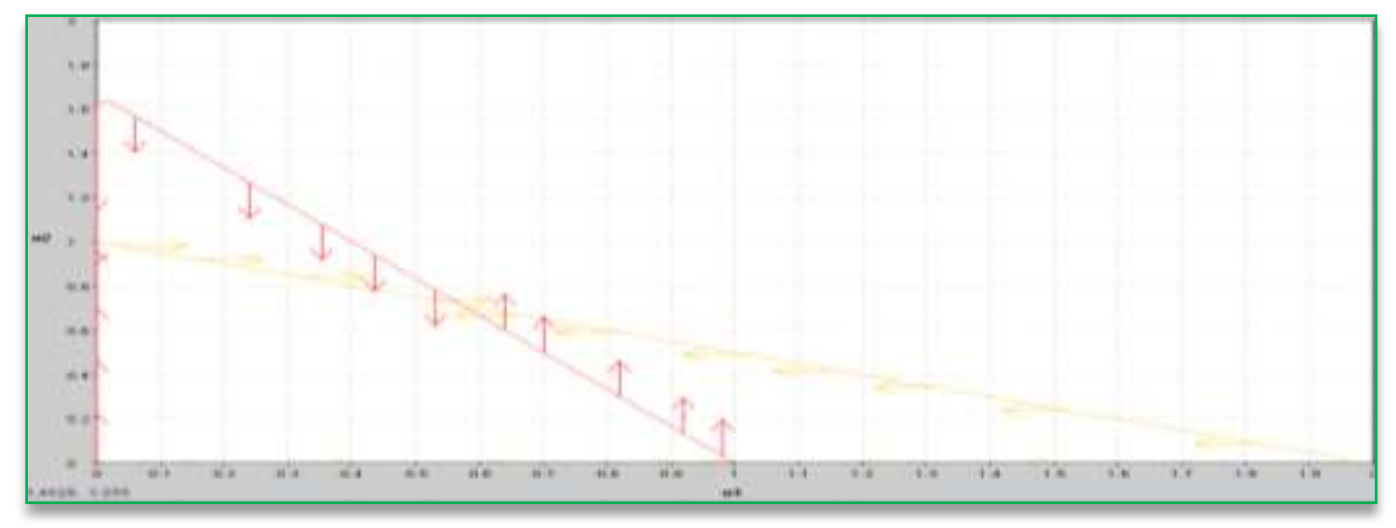

Figure 1.5. The Isoclines for the System $\frac{d u_{1}}{d \tau}=u_{1}\left(1-u_{1}-a_{12} u_{2}\right)$ and $\frac{d u_{2}}{d \tau}=$ $r u_{2}\left(1-u_{2}-a_{21} u_{1}\right)$ for $a_{12}<1$ and $a_{21}<1$

\subsection{Phase Plane Diagram for Logistic Competition Model}

The phase plane portrait sketch for the system:

$$
\begin{aligned}
& \frac{d u_{1}}{d \tau}=u_{1}\left(1-u_{1}-a_{12} u_{2}\right) \\
& \frac{d u_{2}}{d \tau}=r u_{2}\left(1-u_{2}-a_{21} u_{1}\right)
\end{aligned}
$$

Showing the two possible cases is: 


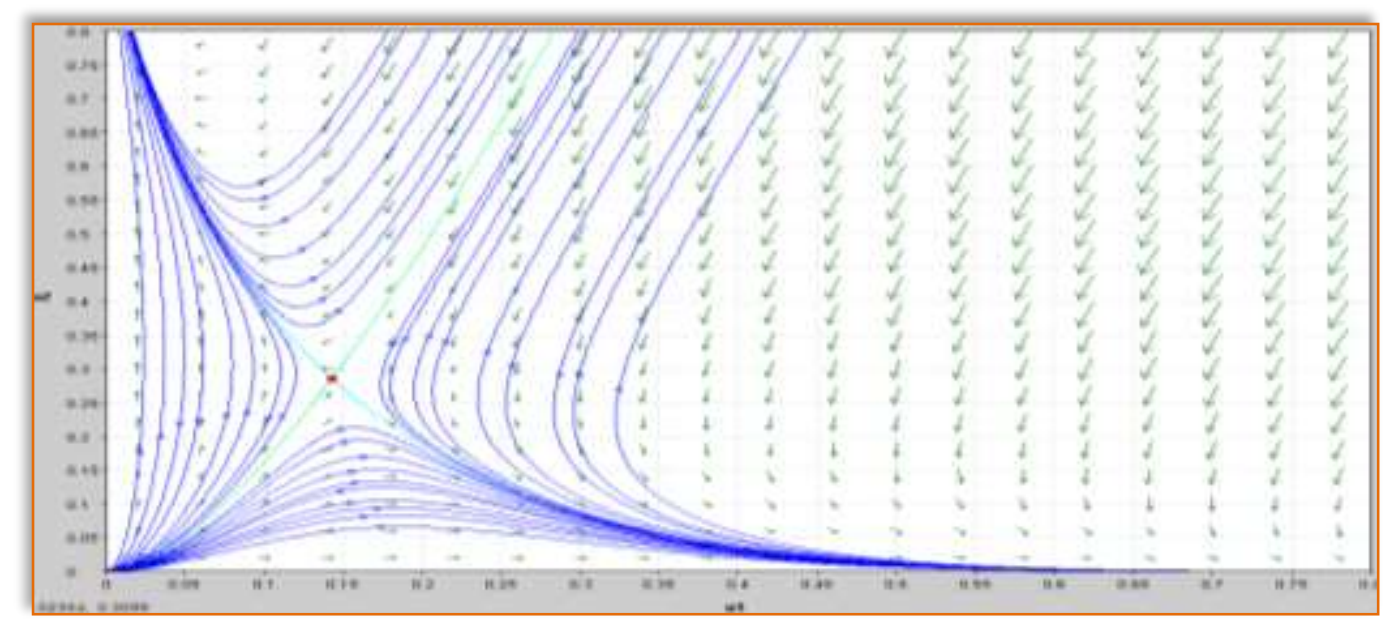

Figure 1.6. The Phase Plane Portrait Sketch for the System $\frac{d u_{1}}{d \tau}=$

$$
u_{1}\left(1-u_{1}-a_{12} u_{2}\right) \text { and } \frac{d u_{2}}{d \tau}=r u_{2}\left(1-u_{2}-a_{21} u_{1}\right)
$$

\subsection{Observation}

Now when we are considering some of the ecological implications of these results, in the case where $a_{12}<1$ and $a_{21}<1$ there is a stable steady state where both species can exist as in Figure 1.5 and in the case where $a_{12}>1$ and $a_{21}>1$ the analysis says that the competition is such that all three nontrivial steady states can exist, but only the steady states $(1,0)$ and $(0,1)$ are stable, as in Figure 1.4.

\section{Type-III}

Both the basic Lotka-Volterra Competition models and its variant with logistic Competition model assume that the two species compete in a linear functional response, $b M_{1}$, which implies that they compete linearly which is not very biologically realistic. Therefore this biologically unrealistic functional response due to Holling (1959), the term $\frac{b M_{1}}{1+b h M_{1}}$ is added and the new competition model is given by

$$
\begin{aligned}
& \frac{d M_{1}}{d t}=r_{1} M_{1}\left(1-\frac{M_{1}}{k_{1}}\right)-\left(\frac{b M_{1}}{1+b h M_{1}}\right) M_{2} \\
& \frac{d M_{2}}{d t}=r_{2} M_{2}\left(1-\frac{M_{2}}{k_{2}}\right)-d M_{1} M_{2}
\end{aligned}
$$

In equation (1.4), the two species $M_{1}$ and $M_{2}$ compete in a Holling type II functional response, $\frac{b M_{1}}{1+b h M_{1}}$.

\subsection{Non-dimensionalization}

To ease calculations and minimize parameters for interpretation, we first set:

Now equation (1.4) can be written as:

$$
M_{1}=M^{*}{ }_{1} \widehat{M}_{1}, \quad M_{2}=M^{*}{ }_{2} \widehat{M}_{2}, \quad t=t^{*} \tau, \quad r=\frac{r_{2}}{r_{1}} \text { and } \tau=\frac{1}{r_{1}}
$$

$$
\begin{aligned}
& \frac{d M^{*}{ }_{1}}{d t^{*}}=\tau r_{1} M^{*}{ }_{1}\left(1-M^{*}{ }_{1}\right)-\frac{\tau k_{2} b M^{*}{ }_{1} M^{*}{ }_{2}}{1+b h M^{*}{ }_{1} k_{1}} \\
& \frac{d M^{*}{ }_{2}}{d t^{*} \tau}=\tau r_{2} M^{*}{ }_{2}\left(1-M^{*}{ }_{2}\right)-\tau d k_{1} M^{*}{ }_{1} M^{*}{ }_{2}
\end{aligned}
$$




$$
\begin{gathered}
\Rightarrow \frac{d M^{*}{ }_{1}}{d t^{*}}=M^{*}{ }_{1}\left(1-M^{*}{ }_{1}\right)-\frac{\left(\frac{k_{2} b}{r_{1}}\right) M^{*}{ }_{1} M^{*}{ }_{2}}{1+b h k_{1} M^{*}{ }_{1}} \\
\frac{d M^{*}{ }_{2}}{d t^{*}}=r M^{*}{ }_{2}\left(1-M^{*}{ }_{2}\right)-\left(\frac{d k_{1}}{r_{1}}\right) M^{*}{ }_{1} M^{*}{ }_{2}
\end{gathered}
$$

Let $\alpha=\frac{k_{2} b}{r_{1}}, \beta=b h k_{1}$ and $\delta=\frac{d k_{1}}{r_{1}}$ where $\alpha, \beta, \delta$ are all positive.

Hence the above equation is minimized to the equation:

$$
\begin{aligned}
& \frac{d M^{*}{ }_{1}}{d t^{*}}=M^{*}{ }_{1}\left(1-M^{*}{ }_{1}\right)-\frac{\alpha M^{*}{ }_{1} M^{*}{ }_{2}}{1+\beta M^{*}{ }_{1}} \\
& \frac{d M^{*}{ }_{2}}{d t^{*} \tau}=r M^{*}{ }_{2}\left(1-M^{*}{ }_{2}\right)-\delta M^{*}{ }_{1} M^{*}{ }_{2}
\end{aligned}
$$

Now removing the asterisks we obtain simple systems of differential equations:

\subsection{Equilibrium Points}

$$
\begin{aligned}
& \frac{d M_{1}}{d t}=M_{1}\left(1-M_{1}\right)-\frac{\alpha M_{1} M_{2}}{1+\beta M_{1}} \\
& \frac{d M_{2}}{d t}=r M_{2}\left(1-M_{2}\right)-\delta M_{1} M_{2}
\end{aligned}
$$

The equilibrium points are $(0,0),(1,0),(0,1)$ and the fourth equilibrium point is obtained by solving the simultaneous equations:

Thus

$$
\begin{aligned}
& M_{1}\left(1-M_{1}\right)-\frac{\alpha M_{1} M_{2}}{1+\beta M_{1}}=0 \\
& r M_{2}\left(1-M_{2}\right)-\delta M_{1} M_{2}=0
\end{aligned}
$$

$(0,0),(1,0),(0,1)$ and $\left(\frac{\alpha \delta+\mathrm{r} \beta-\mathrm{r} \pm \sqrt{(\mathrm{r}-\alpha \delta-\mathrm{r} \beta)^{2}-4 \mathrm{r} \beta(\alpha \mathrm{r}-\mathrm{r})}}{2 \mathrm{r} \beta}, 1-\right.$ $\left.\delta\left(\frac{\alpha \delta+\mathrm{r} \beta-\mathrm{r} \pm \sqrt{(\mathrm{r}-\alpha \delta-\mathrm{r} \beta)^{2}-4 \mathrm{r} \beta(\alpha \mathrm{r}-\mathrm{r})}}{2 \mathrm{r} \beta}\right)\right)$ are the equilibrium points.

3.3. Phase Plane Diagram for Functional Response Type II

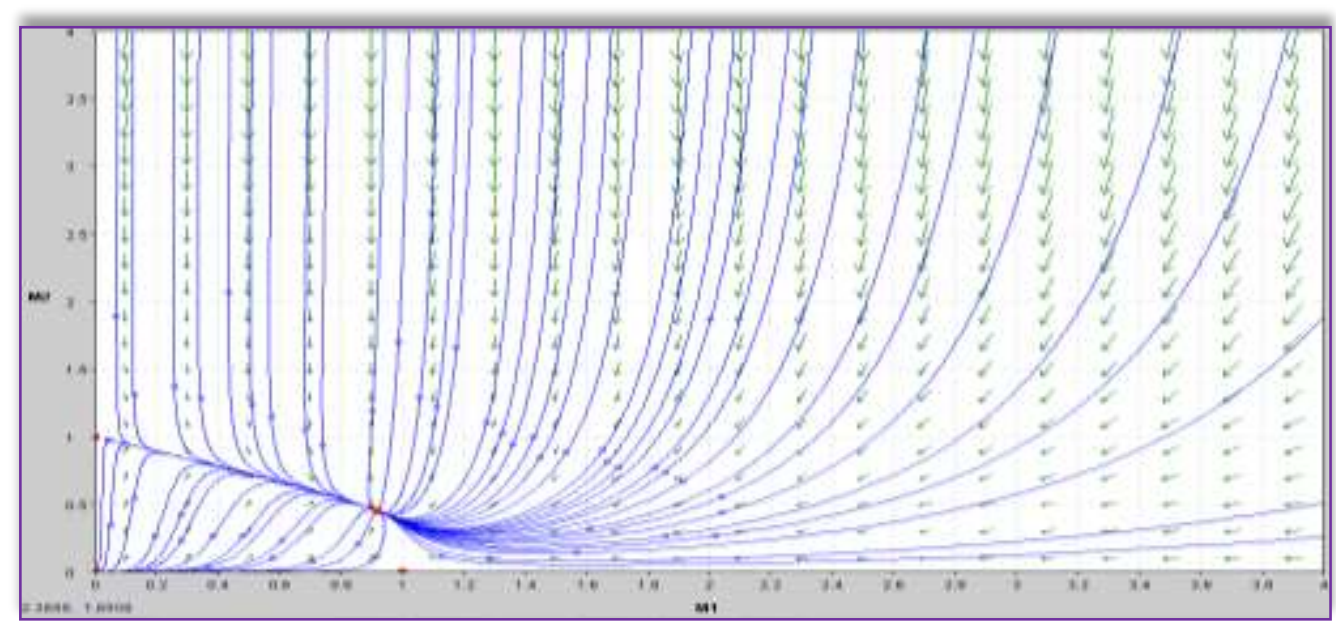

Figure 1.7. Equilibrium Points (Red Dots) and Phase Plane Diagram for the System

$$
\begin{gathered}
\frac{d M_{1}}{d t}=M_{1}\left(1-M_{1}\right)-\frac{\alpha M_{1} M_{2}}{1+\beta M_{1}}, \\
r M_{2}\left(1-M_{2}\right)-\delta M_{1} M_{2}=0
\end{gathered}
$$




\subsection{Nullicline Analysis for Functional Response Type II}

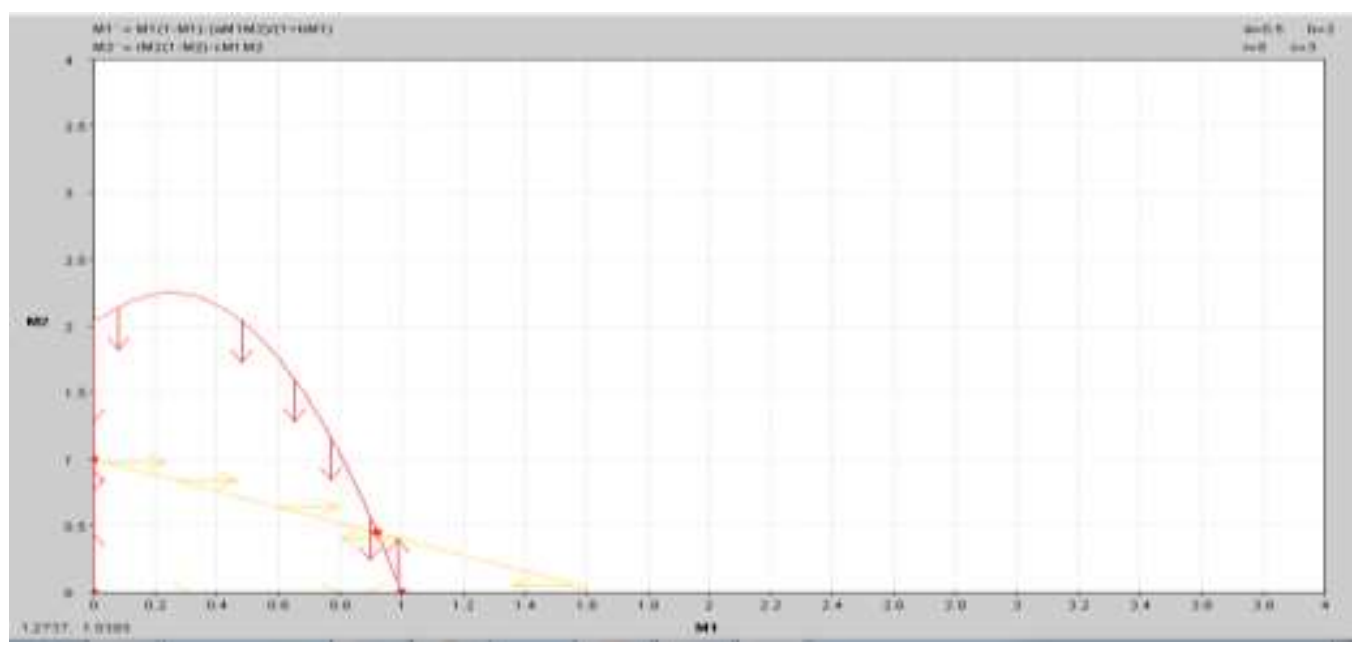

Figure 1.8. Nulliclines for the System $\frac{d M_{1}}{d t}=M_{1}\left(1-M_{1}\right)-\frac{\alpha M_{1} M_{2}}{1+\beta M_{1}}, \frac{d M_{2}}{d t}=r M_{2}(1-$ $\left.M_{2}\right)-\delta M_{1} M_{2}$

\section{Type-IV}

In each of the Competition model above and its variant with logistic Competition model assume that they compete linearly which is not very biologically realistic. Therefore this biologically unrealistic functional response due to Holling type III functional response (1959), the term $\frac{b M_{1}{ }^{2}}{h^{2}+M_{1}{ }^{2}}$ is added which tends to the new competition model given by:

$\frac{d M_{1}}{d t}=r_{1} M_{1}\left(1-\frac{M_{1}}{k_{1}}\right)-\left(\frac{b M_{1}^{2}}{h^{2}+M_{1}^{2}}\right) M_{2}$

$\frac{d M_{2}}{d t}=r_{2} M_{2}\left(1-\frac{M_{2}}{k_{2}}\right)-d M_{1} M_{2}$

In equation (1.5), the two species $\mathrm{M}_{1}$ and $\mathrm{M}_{2}$ compete in a Holling type III functional response, $\frac{\mathrm{bM}_{1}{ }^{2}}{\mathrm{~h}^{2}+\mathrm{M}_{1}{ }^{2}}$.

\subsection{Non-dimensionalization}

To ease calculations and minimize parameters for clear interpretation, we first set:

$$
\mathrm{M}_{1}=\mathrm{M}^{*}{ }_{1} \widehat{\mathrm{M}}_{1}, \quad \mathrm{M}_{2}=\mathrm{M}^{*}{ }_{2} \widehat{\mathrm{M}}_{2}, \quad \mathrm{t}=\mathrm{t}^{*} \tau, \quad \tau=\frac{1}{r_{1}} \text { and } \mathrm{r}=\frac{\mathrm{r}_{2}}{\mathrm{r}_{1}}
$$

Now equation (1.5) can be written as:

where $\widehat{\mathrm{M}}_{1}=\mathrm{k}_{1}$ and $\widehat{\mathrm{M}}_{2}=\mathrm{k}_{2}$

$$
\begin{gathered}
\frac{\mathrm{dM}^{*}{ }_{1}}{\mathrm{dt}^{*} \tau}=\mathrm{r}_{1} \mathrm{M}^{*}{ }_{1}\left(1-\mathrm{M}^{*}{ }_{1}\right)-\left(\frac{\mathrm{bM}^{* 2}{ }_{1} \mathrm{k}_{1}}{\mathrm{~h}^{2}+\mathrm{M}^{* 2}{ }_{1} \mathrm{k}_{1}{ }^{2}}\right) \mathrm{M}_{2}{ }_{2} \mathrm{k}_{2} \\
\frac{\mathrm{dM}^{*}{ }_{2}}{\mathrm{dt}^{*} \tau}=\mathrm{r}_{2} \mathrm{M}^{*}{ }_{2}\left(1-\frac{\mathrm{M}^{*}}{\mathrm{k}_{2}}\right)-\mathrm{dk}_{1} \mathrm{M}^{*}{ }_{1} \mathrm{M}^{*}{ }_{2}
\end{gathered}
$$

Now, we have:

$$
\begin{gathered}
\frac{d M^{*}{ }_{1}}{d t^{*}}=M^{*}{ }_{1}\left(1-M^{*}{ }_{1}\right)-\frac{\mathrm{k}_{1} k_{2} b M^{*}{ }_{1}{ }^{2} M^{*}{ }_{2}}{r_{1}\left(\mathrm{~h}^{2}+\mathrm{M}^{* 2}{ }_{1} \mathrm{k}_{1}{ }^{2}\right)} \\
\frac{d M^{*}{ }_{2}}{d t^{*}}=r M^{*}{ }_{2}\left(1-M^{*}{ }_{2}\right)-\left(\frac{d k_{1}}{r_{1}}\right) M^{*}{ }_{1} M{ }^{*}{ }_{2}
\end{gathered}
$$


Suppose

$$
\alpha=\frac{k_{1} k_{2} b}{r_{1}}, \text { and } \beta=\frac{d k_{1}}{r_{1}} \text { where } \alpha, \beta \text { are all positive constants. }
$$

Hence the above equation is minimized to the equation:

$$
\begin{gathered}
\frac{d M^{*}{ }_{1}}{d t^{*}}=M^{*}{ }_{1}\left(1-M^{*}{ }_{1}\right)-\frac{\alpha M^{*}{ }_{1}{ }^{2} M^{*}{ }_{2}}{\mathrm{~h}^{2}+\mathrm{M}^{*}{ }_{1}{ }_{1} \mathrm{k}_{1}{ }^{2}} \\
\frac{d M^{*}{ }_{2}}{d t^{*}}=r M^{*}{ }_{2}\left(1-M^{*}{ }_{2}\right)-\beta M^{*}{ }_{1} M^{*}{ }_{2}
\end{gathered}
$$

Now removing the asterisks we obtain simple systems of differential equations:

\subsection{Equilibrium Points}

$$
\begin{gathered}
\frac{d M_{1}}{d t}=M_{1}\left(1-M_{1}-\frac{\alpha M_{1} M_{2}}{\mathrm{~h}^{2}+{M_{1}}^{2} \mathrm{k}_{1}{ }^{2}}\right) \\
\frac{d M_{2}}{d t}=r M_{2}\left(1-M_{2}-\beta M_{1}\right)
\end{gathered}
$$

The equilibrium points are $(0,0),(1,0),(0,1)$ and the other equilibrium points are obtained by solving the simultaneous equation:

$$
\begin{gathered}
M_{1}+\frac{\alpha M_{1} M_{2}}{\mathrm{~h}^{2}+M_{1}{ }^{2} \mathrm{k}_{1}{ }^{2}}=1 \\
\beta M_{1}+M_{2}=1
\end{gathered}
$$

From $\beta M_{1}+M_{2}=1$ we have:

$$
M_{1}=\frac{1-M_{2}}{\beta}
$$

By substituting $M_{1}=\frac{1-M_{2}}{\beta}$ in the equation $M_{1}+\frac{\alpha M_{1} M_{2}}{\mathrm{~h}^{2}+M_{1}{ }^{2} \mathrm{k}_{1}{ }^{2}}=1$ we get an equation of the form in the variable $M_{2}$ :

$$
\begin{gathered}
{k_{1}}^{2} M_{2}{ }^{3}+\left(\beta k_{1}{ }^{2}-3{k_{1}}^{2}\right) M_{2}{ }^{2}+\left(3 k_{1}{ }^{2}+\beta^{2} h^{2}-2 \beta k_{1}{ }^{2}\right) M_{2} \\
+\left(\beta^{3} h^{2}+\beta k_{1}{ }^{2}-k_{1}{ }^{2}-\beta^{2} h^{2}\right)=0
\end{gathered}
$$

This has at most three roots. Therefore the system

$$
\begin{gathered}
\frac{d M_{1}}{d t}=M_{1}\left(1-M_{1}-\frac{\alpha M_{1} M_{2}}{\mathrm{~h}^{2}+M_{1}{ }^{2} \mathrm{k}_{1}{ }^{2}}\right) \\
\frac{d M_{2}}{d t}=r M_{2}\left(1-M_{2}-\beta M_{1}\right)
\end{gathered}
$$

has four equilibrium points which are shown below on the phase portrait diagram.

\subsection{Equilibrium Points Diagram for Functional Response Type III}

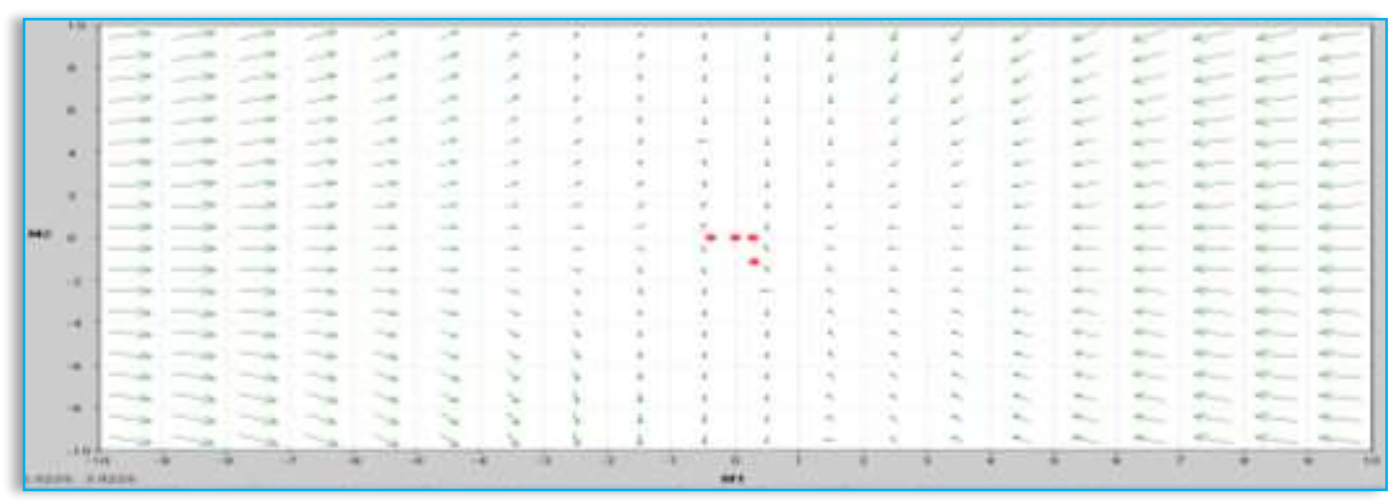

Figure 1.9. Equilibrium Points (Red Dots) for the System

$$
\frac{d M_{1}}{d t}=M_{1}\left(1-M_{1}-\frac{\alpha M_{1} M_{2}}{\mathrm{~h}^{2}+M_{1}{ }^{2} \mathrm{k}_{1}{ }^{2}}\right)
$$




$$
\frac{d M_{2}}{d t}=r M_{2}\left(1-M_{2}-\beta M_{1}\right)
$$

\subsection{Phase Plane Diagram for Functional Response Type III}

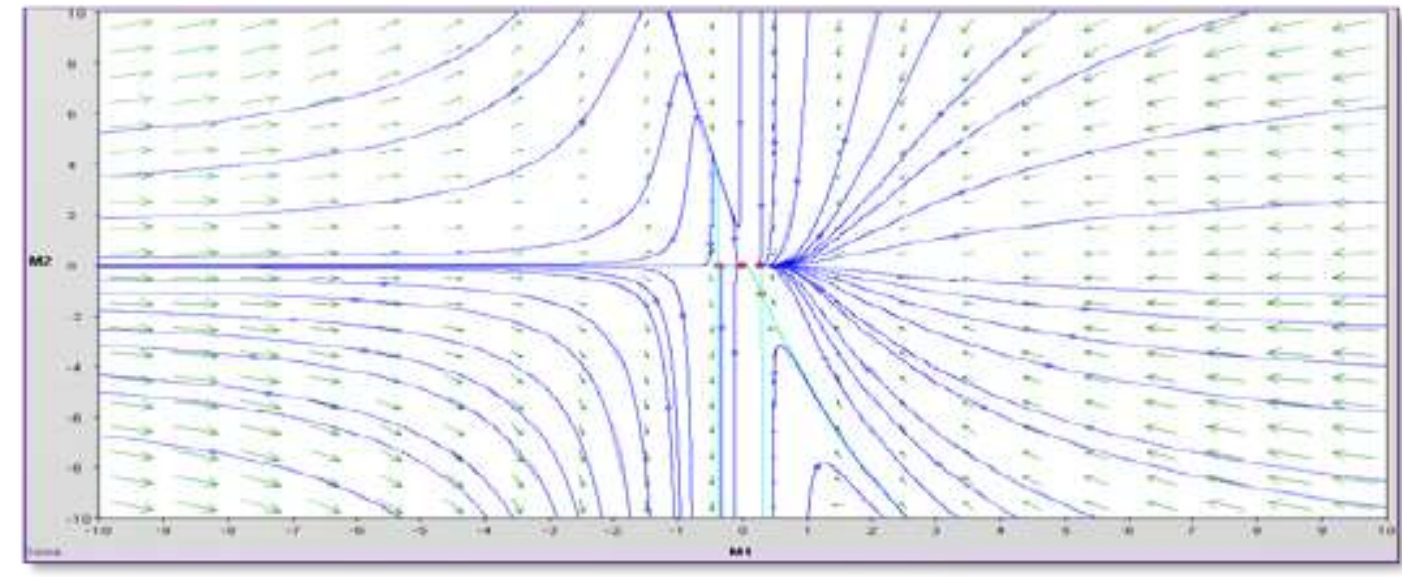

Figure 1.10. Phase Plane Diagram for the System

$$
\frac{d M_{1}}{d t}=M_{1}\left(1-M_{1}-\frac{\alpha M_{1} M_{2}}{\mathrm{~h}^{2}+M_{1}{ }^{2} \mathrm{k}_{1}^{2}}\right), \frac{d M_{2}}{d t}=r M_{2}\left(1-M_{2}-\beta M_{1}\right)
$$

\section{Conclusions}

(i).The Lotka-Volterra model of interspecific competition has been a useful starting point for biologists thinking about the outcomes of competitive interactions between species. The assumptions of the model (e.g., there can be no migration and the carrying capacities and competition coefficients for both species are constants) may not be very realistic, but are necessary simplifications.

(ii).A variety of factors not included in the model can affect the outcome of competitive interactions by affecting the dynamics of one or both populations. Environmental change, disease, and chance are just a few of these factors.

\section{Acknowledgement}

The authors are grateful to SERSC for their constant encouragement who are extending their cooperation with financial support for the articles which are published in IJBSBT.

\section{References}

[1] H. Weiss, "A Mathematical Introduction to Population Dynamics, Georgia, USA, (2010).

[2] J. D. Murray, "Mathematical Models in life sciences", John Wiley and Sons, (2010).

[3] P.Waltman, "Competition Models in Population Biology", Emory University, Atlanta, Georgia, (1983).

[4] S. B. Hsu and T. W. Hwang, "Global stability for a class of Predator-Prey System", (1995).

[5] F. Verhulst, "Nonlinear differential equations and dynamical systems", Second edition, Springer-Verlag, Berlin, (1996).

[6] W.E. Boyce; R.C. Diprima, "Elementary Differential Equations and Boundary Value Problems (4th ed.)", John Wiley and Sons, (1986). 